\title{
Bletilla striata polysaccharide modified collagen fiber composite sponge with rapid hemostasis function
}

Xingyu Yan ${ }^{1,2,3}$, Yining Chen ${ }^{1,2,3}$, Weihua Dan ${ }^{1,2,3}$, Nianhua Dan ${ }^{1,2,3^{*}}$ and Zhengjun Li $\mathrm{i}^{1,2,3}$

\begin{abstract}
Emergencies often result in uncontrollable bleeding, which is thought to be the leading cause of death at the scene of the injured. Among various hemostasis scenarios, collagen fiber (CF) is gradually replacing traditional hemostatic materials due to its superior properties and ease of sourcing from animals. Herein, we use CF and the natural herbaceous Bletilla striata as raw materials to prepare a collagen fiber-oxidized Bletilla striata composite hemostatic sponge (CFOB). During the cross-linking process, the triple helix structure of collagen stays intact, and its porous threedimensional network structure brings excellent bulkiness and water absorption properties. Experiments show that the optimal amount of sponge CFOB-10, namely oxidized Bletilla striata polysaccharide $0.5 \mathrm{mg} / \mathrm{mL}$ and CF $5 \mathrm{mg} / \mathrm{mL}$, only needed $25 \pm 4.06 \mathrm{~s}$ for hemostasis time in the rat liver hemorrhage model. In addition, CFOB meets the safety performance requirements of cytotoxicity classification standard 0 . Therefore, the optimal amount of CFOB is an excellent new hemostatic material with application potential.
\end{abstract}

Keywords Wound hemostasis, Collagen fiber, Bletilla striata polysaccharide

${ }^{*}$ Correspondence:

Nianhua Dan

dannianhua@scu.edu.cn

Full list of author information is available at the end of the article

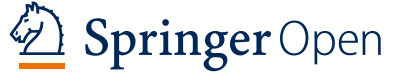

C The Author(s) 2022, corrected publication 2023. Open Access This article is licensed under a Creative Commons Attribution 4.0 International License, which permits use, sharing, adaptation, distribution and reproduction in any medium or format, as long as you give appropriate credit to the original author(s) and the source, provide a link to the Creative Commons licence, and indicate if changes were made. The images or other third party material in this article are included in the article's Creative Commons licence, unless indicated otherwise in a credit line to the material. If material is not included in the article's Creative Commons licence and your intended use is not permitted by statutory regulation or exceeds the permitted use, you will need to obtain permission directly from the copyright holder. To view a copy of this licence, visit http://creativecommons.org/licenses/by/4.0/. 


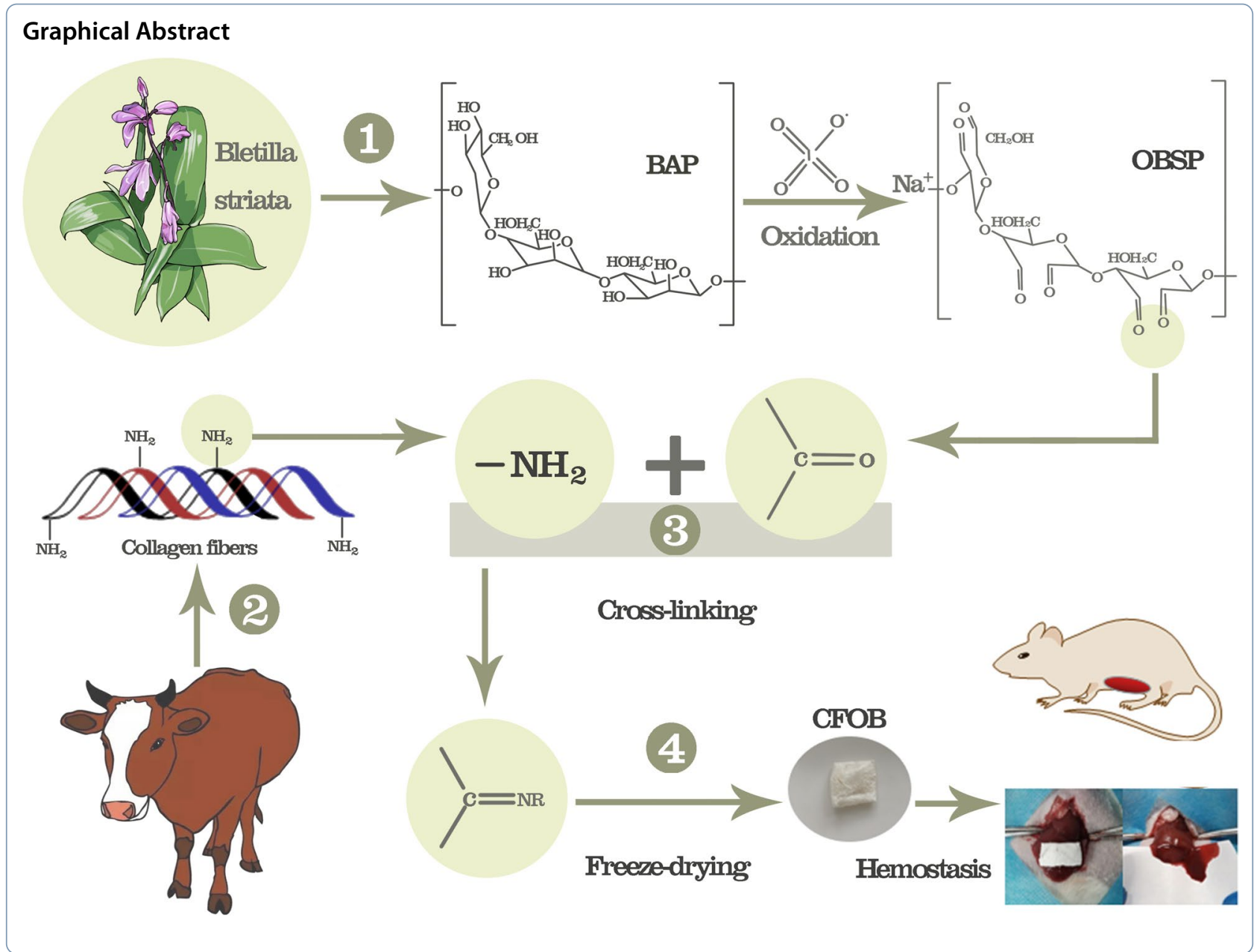

\section{Introduction}

Emergency accidents that occur in various situations, such as car accidents and warfare, are often difficult to control. According to multidisciplinary statistics, about 39\% of the deaths in accidents are caused by bleeding, so wound bleeding is also considered to be the leading cause of death at the scene [1]. Thus, the quick and effectual hemostasis of the wounded is significant. Shortening the time and improving the quality of hemostasis may be the best strategy to reduce the mortality of the wounded. At present, in-depth researches on various hemostatic materials have been widely carried out [2]. Among them, collagen stands out for its superior performance, which has gradually been attracting the attention of researchers.

Collagen is readily available and abundant in animal bodies. With hemostatic properties, superior biocompatibility, low antigenicity, and degradability, collagen has gradually replaced traditional hemostatic materials, applied to various hemostatic scenarios [3, 4]. Collagen fiber (CF) is a fiber woven fabric formed by stacking, gathering, arranging, and entwining collagen molecules, and the hemostatic performance of CF is enhanced with the increasing number of layers. Compared with collagen, although CF performs better in hemostasis, pure collagen materials can only meet the basic requirements of hemostatic agents and cannot be applied to more complex conditions.

Unlike other cross-linking agents with cytotoxicity and inflammation, natural hemostatic derivatives show good biocompatibility and absorbable degradability. At the same time, the extraction technology of natural products' effective components is becoming more mature.

Bletilla striata is a kind of terrestrial herbaceous plant belonging to Orchidaceae. Bletilla striata polysaccharide (BSP), a natural macromolecular polysaccharide with a major component of mannose and glucose (ratio of 1 : 4), an average molecular weight of around 2.8 million, is the active component in Bletilla striata. BSP may be employed as a range of drug-loaded dressings and biological hemostatic materials because of its high hemostatic capabilities and great biocompatibility. Zhang and 
colleagues created a BSP hydrogel using BSP [5], and their results showed that the BSP hydrogel not only had hemostatic function but also promoted wound repair and inhibits scar formation. Zhao and coworkers modified BSP with stearic acid to form micelles of tumor-targeted drugs [6], and analysis results showed that this micelle was $\mathrm{pH}$-sensitive and had the potential to become a drug carrier for anti-rabies.

To further improve the properties of collagen materials, particularly the hemostatic performance, we extracted the active ingredient BSP and selectively oxidized it into oxidized Bletilla striata polysaccharide (OBSP). Then, OBSP was used to modify the CF, and finally, a hemostatic sponge compounded with collagen fiber (CFOB) was obtained, and its related properties were studied.

\section{Experiments and methods}

\subsection{Experimental instruments and equipment}

GL-20G-II high-speed refrigerated centrifuge, Shanghai Anting Scientific Instrument Factory; Freeze6 refrigerated dryer, Labconco, USA; EA1004 high-precision electronic balance, Beijing Sartorius Co., Ltd.; SK5200H ultrasonic cleaner, Shanghai Branch Guide Instrument Co., Ltd.; 200SXV-IR Fourier Infrared Spectrometer, Nicolet, USA; DSC Differential Scanning Calorimeter, GE, USA; Model 400 Circular Dichromatometer, AVAV, USA; Model S520 Scanning Electron Microscope, Japan Electronics Co., Ltd.; SHB-III circulating water multipurpose vacuum pump, Zhengzhou Dufu Instrument Factory.

\subsection{Main reagents and materials}

Medical grade Bletilla striata, Western Biotech; Fresh bovine Achilles tendon; filtered pure calf serum (BCS), Hyclone; AR absolute ethanol, Chengdu Jinshan Reagent Co., Ltd.; AR sodium citrate, Chengdu Jinshan Reagent Co., Ltd.; AR glacial acetic acid, Chengdu Jinshan Reagent Co., Ltd.; AR RPMI1640 medium, Gibcobrl, USA; AR CCK-8, Sigma, USA; AR Papain, Chengdu Kelon Chemical Reagent Factory; AR Lead Nitrate, Chengdu Jinshan Reagent Co., Ltd.

\subsection{Preparation method}

\subsubsection{Extraction of $C F$}

CF was extracted according to the literature [7].

\subsubsection{Purification of Bletilla striata polysaccharide (BSP)}

A specific amount of Bletilla powder was accurately weighed at room temperature and dissolved in deionized water with a liquid ratio of 1:50. To thoroughly dissolve the solution, it was magnetically agitated for $1 \mathrm{~h}$. A specific amount of papain was accurately weighed and added to the Bletilla striata crude polysaccharide solution and stirred magnetically for $1 \mathrm{~h}$. After thoroughly dissolving the solution, $1 \mathrm{~mol} / \mathrm{L} \mathrm{NaOH}$ was added to retain the $\mathrm{pH}$ at 7 , and the solution was enzymatically hydrolyzed at $48{ }^{\circ} \mathrm{C}$ for $120 \mathrm{~min}$. Anhydrous ethanol was added to the solution after the reaction at a liquid ratio of 1:9 to precipitate the BSP in a flocculent condition. After centrifugation at $8000 \mathrm{r} / \mathrm{min}$ for $5 \mathrm{~min}$, the precipitate was collected and redissolved in water. During this period, the insoluble matter was removed by suction filtration. The collected filtrate was centrifugated again at 8000r/ min for repeating 4-5 times. The final product was dried to constant weight in a constant temperature drying oven to obtain purified BSP [8].

\subsubsection{Preparation of OBSP}

An appropriate amount of purified BSP was taken and dissolved in deionized water with a liquid ratio of 1:50. Sodium periodate was added in a ratio of $1: 1.3$, and wrapped with tin foil to protect it from light, and oxidized under magnetic stirring for $24 \mathrm{~h}$ at room temperature. After the reaction, ethylene glycol was added and reacted for $0.5 \mathrm{~h}$, and the product was put into a dialysis bag, dialyzed in distilled water for three days and freezedried to obtain OBSP.

\subsubsection{Preparation of CFOB}

Separately, CF and OBSP were dissolved in $0.5 \mathrm{~mol} / \mathrm{L}$ of acetic acid and prepared into $5 \mathrm{mg} / \mathrm{ml}$ solutions. OBSP solution dropwise was added to the CF solution so that the amount of OBSP in varying samples was $2 \%, 6 \%$, $10 \%, 15 \%, 20 \%$ of the CF content respectively, and they were stirred slowly for $24 \mathrm{~h}$ at a constant temperature of $4{ }^{\circ} \mathrm{C}$. After freeze-drying, the $\mathrm{CFOB}$ was obtained, which were denoted as CFOB-0, CFOB-2, CFOB-6, CFOB-10, CFOB-15, and CFOB-20.

\subsection{Characterization method}

2.4.1 Fourier-transform infrared (FT-IR)

$1 \mathrm{mg}$ CFOB, $1 \mathrm{mg}$ BSP, and $1 \mathrm{mg}$ OBSP were scraped into the mortars successively, and separately $100 \mathrm{mg}$ of potassium bromide $(\mathrm{KBr})$ were added to obtain FT-IR spectra of them on an FTIR spectrophotometer. The transmission mode was $4 \mathrm{~cm}^{-1}$ intervals and within the wavelength range of $4000-400 \mathrm{~cm}^{-1}$ in a dry atmosphere. Relative humidity was less than $65 \%$, at room temperature $\left(23 \pm 1{ }^{\circ} \mathrm{C}\right)$, and scanning was repeated 32 times.

\subsubsection{Circular dichroism (CD) spectral analysis}

Diluted the CFOB solution to $0.1 \mathrm{mg} / \mathrm{mL}$. The solution was injected into a quartz cell with a $1 \mathrm{~mm}$ optical path, and the solution was scanned using a Circular Dichroism Spectrometer. The scanning range was $190-260 \mathrm{~nm}$, 
and scanning rate was $100 \mathrm{~nm} / \mathrm{min}$, and scanning was repeated 3 times.

\subsubsection{Differential scanning calorimetry (DSC)}

Differential scanning calorimetry (DSC) can detect the heat flow difference between the sample and the reference during the process of temperature increase, characterizing the thermal stability of the sample on the Differential scanning calorimeter. Six groups of CFOB samples $(2 \mathrm{mg})$ were respectively taken and sealed in a DSC crucible. An empty crucible was used as a reference, and nitrogen was used as the protective gas in the sample chamber. The temperature was heated from $20^{\circ} \mathrm{C}$ to $180{ }^{\circ} \mathrm{C}$ at the speed of $10{ }^{\circ} \mathrm{C} \mathrm{min}^{-1}$.

\subsubsection{Atomic force microscopy (AFM) and scanning electron microscopy (SEM)}

CFOB was cut into small pieces with a thickness of 9 um by frozen sectioning. Then atomic force microscopy was used to analyze the samples at a fixed scanning rate of $1 \mathrm{~Hz}$ at room temperature.

The morphology of the different amounts of CFOB was observed using a scanning electron microscope. All samples were sputter-coated with gold and imaged at an accelerating voltage of $5 \mathrm{kV}$.

\subsubsection{Porosity ratio determination}

An ethanol substitution method was used to test the porosity of sponges [9]. A preweighed empty bottle $\left(M_{1}\right)$ was first filled with ethanol, then measured $\left(\mathrm{M}_{2}\right)$. Then, the pre-weighed CFOB $\left(\mathrm{M}_{\mathrm{s}}\right)$ was put to the bottle, where some ethanol spilled out of the bottle and treated with ultrasound for $10 \mathrm{~min}$. Afterward, ethanol was added into the bottle until it was filled with ethanol again and measured $\left(\mathrm{M}_{3}\right)$. Finally, the ethanol in the bottle was drained and residual ethanol was absorbed by cotton swabs, before weighing the bottle and CFOB $\left(\mathrm{M}_{4}\right)$. Averaging three measurements were performed. Formula 1 is used to calculate porosity (P).

$$
\mathrm{P}=\left(\mathrm{M}_{4}-\mathrm{M}_{1}-\mathrm{M}_{\mathrm{s}}\right) /\left(\mathrm{M}_{4}-\mathrm{M}_{1}+\mathrm{M}_{2}-\mathrm{M}_{3}\right) \times 100
$$

\subsubsection{Determination of water absorption}

To measure the water absorption ratios, CFOB were cut into the same size of $2 \mathrm{~cm}: 2 \mathrm{~cm}$ square. Then the preweighed sponge $\left(\mathrm{W}_{1}\right)$ was put into one beaker filled with abundant normal saline solution. After CFOB absorbed liquid to saturation, it was taken out using tweezers and suspended in the air for $1 \mathrm{~min}$. Finally, the sponge with effectively absorbed liquid was weighed $\left(\mathrm{W}_{2}\right)$ and captured using a digital camera. The experiment was repeated at least three times. The calculation Formula 2 of water absorption (W) is:

$$
\mathrm{W}=\left(\mathrm{W}_{2}-\mathrm{W}_{1}\right) / \mathrm{W}_{1} \times 100
$$

\subsubsection{Hemolysis assay}

The experiment was carried out according to the reported hemolysis assay method [10]. The solution with different amounts of CFOB were prepared, which was mixed with $5 \mathrm{~mL}$ of diluted blood, which was normal saline $0.9 \%$ $\mathrm{NaCl}$ containing $2 \%$ rat blood. After incubation at $37{ }^{\circ} \mathrm{C}$ for $60 \mathrm{~min}$, the blood cell suspension was centrifuged at $1000 \mathrm{rpm}$ for $10 \mathrm{~min}$, and then the hemolysis rate was measured at $545 \mathrm{~nm}$ by ultraviolet spectrophotometer. $0.2 \mathrm{~mL}$ of diluted blood with $10 \mathrm{~mL}$ deionized water was performed as the positive control group (100\% hemolysis), whereas, $0.2 \mathrm{~mL}$ of diluted blood with $10 \mathrm{~mL}$ normal saline was regarded as the negative control group ( $0 \%$ hemolysis). The calculation Formula 3 of hemolysis rate $(\mathrm{X})$ is as follows:

$$
\mathrm{X}=(\mathrm{A}-\mathrm{B}) /(\mathrm{C}-\mathrm{B}) \times 100
$$

In the formula: A means absorbance of the experimental sample; B means absorbance of the negative control; C means absorbance of the positive control.

\subsubsection{Determinations of activated partially thromboplastin time (APTT), prothrombin time (PT) and thrombin time (TT)}

According to the practice of Eren [11], the APTT, PT and, TT were evaluated in vitro. Mix fresh rat blood with a $3.8 \%(\mathrm{v} / \mathrm{v})$ sodium citrate solution in a volume ratio of 1:9. The anticoagulated rat blood was centrifugated at $1500 \mathrm{r} / \mathrm{min}$ for $15 \mathrm{~min}$ at $37^{\circ} \mathrm{C}$ to obtain platelet-poor plasma (PPP). Subsequently, $0.2 \mathrm{~g}$ of CFOB samples with different contents were crushed and dispersed in $100 \mathrm{~mL}$ of physiological saline to prepare a physiological saline suspension with a sample concentration of $0.2 \mathrm{mg} / \mathrm{ml}$. $0.5 \mathrm{~mL}$ of the sample physiological saline suspension was mixed with $1 \mathrm{ml}$ of the above platelet-poor plasma and shaken. Then Actin reagent, Thromborel S reagent and, Thrombin reagent were added respectively to the suspension of samples with different contents. With normal saline as a blank control group, the APTT, PT and, TT of each sample were measured on an automatic coagulometer.

\subsubsection{Whole-blood clotting assay}

$0.02 \mathrm{~g}$ each of CFOB in different dosages was taken in a test tube, $0.02 \mathrm{~mL}$ of $0.025 \mathrm{~mol} / \mathrm{L}$ calcium chloride and $0.2 \mathrm{~mL}$ of fresh rat blood were added in the test tube and mixed after 20 min of incubation in a $37^{\circ} \mathrm{C}$ water bath. After mixing, samples were put in a $37{ }^{\circ} \mathrm{C}$ water bath 
again and started recording the time with a stopwatch until the blood was completely coagulated. 3 parallel samples were tested in each group and the average value was taken.

\subsubsection{Blood clotting index ( $B C l)$}

The coagulation properties in vitro of materials are often evaluated by the blood clotting index (BCI), according to the report [12]. $0.1 \mathrm{~mL}$ fresh blood was dropped into $0.5 \mathrm{~cm}^{2}$ sample placed in a beaker at $37{ }^{\circ} \mathrm{C} .0 .02 \mathrm{~mL}$ of $0.2 \mathrm{~mol} / \mathrm{L} \mathrm{CaCl}{ }_{2}$ solutions were added and incubated for $5 \mathrm{~min}$ at room temperature, and then the absorption and coagulation promoting effect of different sample materials on blood were observed. After adding $25 \mathrm{~mL}$ of ultrapure water and shaking slightly for $5 \mathrm{~min}$, the mixture solution was analyzed at wavelength $545 \mathrm{~nm}$ for the ultraviolet absorbance test. $0.1 \mathrm{~mL}$ of blood with $25 \mathrm{~mL}$ of ultrapure water was set as the control group. The absorbance of the sample was recorded as $\mathrm{Abs}_{\mathrm{s}}$, and the absorbance of the blank group was $\mathrm{Abs}_{0}$, then the blood clotting index (BCI) can be calculated by Formula 4:

$$
\mathrm{BCI}=100 \times\left(\mathrm{Abs}_{\mathrm{s}}\right) /\left(\mathrm{Abs}_{0}\right)
$$

\subsubsection{Interaction between blood cells and the materials}

According to the literature [10], the interaction between the surface of materials and blood cells, mainly platelets and red blood cells, was observed via SEM. $0.1 \mathrm{~mL}$ fresh blood containing anticoagulant (sodium citrate) was added to CFOB $\left(1 \times 1 \times 0.5 \mathrm{~cm}^{3}\right)$. After incubation at $37^{\circ} \mathrm{C}$ for $1 \mathrm{~min}, 3 \mathrm{~min}$, and $5 \mathrm{~min}$ respectively, then washing physical adhesion cells with PBS buffer for $10 \mathrm{~min}$ and three times, the cleaned sample composite was fixed with $2.5 \%$ glutaraldehyde, dehydrated with ethanol, and finally, freeze-dried. The adhesion of blood cells on the material was observed by SEM.

\subsubsection{Evaluation of cell compatibility}

The fibroblasts (L929) cultured to the logarithmic phase were used as model cells, and the cytocompatibility of CFOB was evaluated by the CCK- 8 method. Before cell inoculation, $\mathrm{CFOB}$ samples were sterilized in an autoclave at $120^{\circ} \mathrm{C}$ for $30 \mathrm{~min}$. Then, it was transferred to the sample in a 96-well plate to dispense a cell suspension $(200 \mu \mathrm{L})$ with a density of $5 \times 10^{4}$ cells $/ \mathrm{mL}$. After $24 \mathrm{~h}$, the CFOB sample was transferred to another 96-well plate, and $200 \mu \mathrm{L}$ of fresh medium, and $20 \mu \mathrm{L}$ of Cell Counting Kit-8 (CCK-8, Dojindo Laboratories, Kumamoto, Japan) solution were added, and then incubated at $37^{\circ} \mathrm{C}$ for $3 \mathrm{~h}$. Finally, $100 \mu \mathrm{L}$ of the medium was distributed to another 96-well plate, and a spectrophotometer was used to measure the formazan dye colorimetrically, and the OD reading was recorded.

\subsubsection{Evaluation of in vivo hemostatic performance}

A hemostasis test was carried out on the rat liver hemorrhage model suffered from scratch on the left lobe of the liver (length $1.0 \mathrm{~cm} \times$ depth $0.5 \mathrm{~cm} \times$ width $0.2 \mathrm{~cm}$ ) by scalpel. After bleeding, the wound was covered with hemostatic materials to stanch bleeding, including $\mathrm{CFOB}, \mathrm{CF}$ sponge, and medical gauze, respectively. Hemostatic time and blood loss, were recorded. Medical gauze was used as the negative control, and an unmodified CF sponge was used as the positive control.

\section{Results}

\subsection{FT-IR spectra measurements}

To investigate the specific information of the molecular structure of BSP after oxidation, a Fourier transform infrared spectrometer was used to detect the molecular structure of BSP and OBSP. As shown in Fig. 1a, the infrared spectrum of BSP shows that it has the absorption peak of polysaccharides. The absorption peak between $3600 \mathrm{~cm}^{-1}$ and $3200 \mathrm{~cm}^{-1}$ is $\mathrm{O}-\mathrm{H}$ stretching vibration, and the absorption peak between $3000 \mathrm{~cm}^{-1}$ and $2750 \mathrm{~cm}^{-1}$ is $\mathrm{C}-\mathrm{H}$ stretching vibration in $-\mathrm{CH}_{2}-$ or $-\mathrm{CH}_{3}$. The absorption peak between $1725 \mathrm{~cm}^{-1}$ and $1500 \mathrm{~cm}^{-1}$ indicates the presence of acyl groups, and the absorption peak at $1380 \mathrm{~cm}^{-1}$ is the bending vibration of $\mathrm{C}-\mathrm{H}$ in $-\mathrm{CH}\left(\mathrm{CH}_{3}\right)_{2}$. The absorption peaks between $1063 \mathrm{~cm}^{-1}$ and $1032 \mathrm{~cm}^{-1}$ and at $920 \mathrm{~cm}^{-1}$ indicate the pyranosylation of BSP, and $810 \mathrm{~cm}^{-1}$ and $870 \mathrm{~cm}^{-1}$ are the characteristic absorption peaks of mannose. Compared with BSP, the increased absorption peak of OBSP at $1729 \mathrm{~cm}^{-1}$ is attributed to the characteristic absorption peak after the o-dihydroxy is oxidized to dialdehyde [13].

The triple helix structure of collagen is particularly important for its maintenance of functional properties. The characteristic absorption peaks of amide A, B, I, II, and III bands can reflect the triple helix structure of natural type I collagen to a certain extent $[14,15]$. It can be seen from Fig. $1 \mathrm{~b}$ that the absorption peaks of the amide band I and band II appear at $1654 \mathrm{~cm}^{-1}$ and $1544 \mathrm{~cm}^{-1}$, respectively. This is due to the synergy of $\mathrm{C}=\mathrm{O}$ stretching vibration, $\mathrm{N}-\mathrm{H}$ bending vibration, and $\mathrm{C}-\mathrm{N}$ stretching vibration in the collagen molecular skeleton. The amide III band appears at about a $1236 \mathrm{~cm}^{-1}$ absorption peak. This is caused by the $\mathrm{C}-\mathrm{N}$ stretching of the amide bond in the collagen molecular chain, the $\mathrm{N}-\mathrm{H}$ in-plane bending vibration, and the swing vibration of the main chain $-\mathrm{CH}_{2}$ group and the side chain glycine and proline. The absorption peaks of the amide $\mathrm{A}$ band and the amide $\mathrm{B}$ band appear at about $3342 \mathrm{~cm}^{-1}$ and $2965 \mathrm{~cm}^{-1}$, respectively. This is due to the characteristic peaks of the $-\mathrm{OH}$ and $-\mathrm{NH}_{2}$ groups of the molecular side chain, which are 

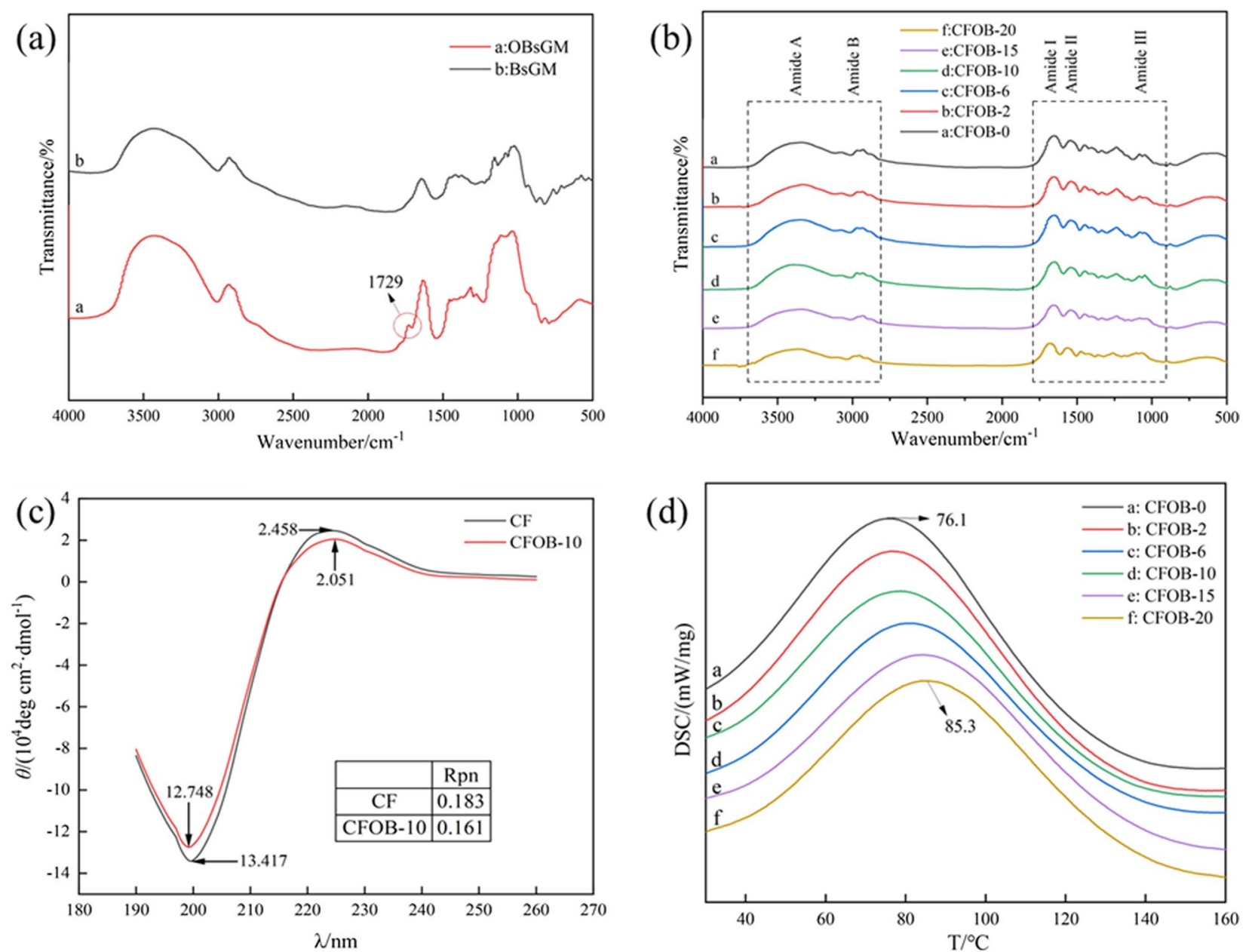

Fig. 1 a Infrared spectra of BSP and OBSP, $\mathbf{b}$ infrared spectrum detection diagram of CFOB with different OBSP dosages, c CD spectral analysis diagram of BSP and OBSP, $\mathbf{d}$ DSC detection chart of CFOB with different OBSP dosages

mainly caused by the stretching vibration of $\mathrm{N}-\mathrm{H}$. The infrared spectrum of CFOB still retains the five characteristic absorption peaks of collagen, which shows that CFOB has not lost the natural triple helix structure, so its biological activity will not be affected. It is not difficult to find from Fig. 1a that after the introduction of OBSP, the absorption peaks of the amide I, II, and III bands of the CF decreased slightly. This indicates that the Schiff base cross-linking reaction of OBSP and CF formed a more stable $\mathrm{C}=\mathrm{N}$ cross-linked bond, which has a certain destructive effect on the hydrogen bond between the CF molecular chains. OBSP with a flexible molecular chain can form multi-molecular scale cross-links with CF molecules, and more adjacent CF molecules can be covalently bonded through Schiff bases to enhance the structural stability of CF molecules. This was also confirmed in subsequent DSC tests. It is worth noting that after the OBSP cross-linked modified CF, the peak shapes of the amide A band and the amide B band broadened to varying degrees, and a slight blue shift occurred. This means that collagen molecules may have self-aggregated to a certain extent [16].

\subsection{CD spectral analysis}

The circular dichroism (CD) detection can more accurately analyze the three-dimensional structure of $\alpha$-helix, $\beta$-sheet, $\beta$-turn, and random coil in the secondary structure of collagen (Fig. 1c). Collagen generally has a strong negative absorption peak around the wavelength of $197 \mathrm{~nm}$, which is mainly caused by the transition of the formula $-r$ amide bond. The strong positive absorption peak around the wavelength of $225 \mathrm{~nm}$ is caused by the transition of the n-r amide bond. In circular dichroism detection, the ratio of the positive peak intensity to the negative peak intensity (Rpn) is usually used to characterize the conformation of the collagen 
solution. When the collagen triple helix structure is significantly damaged, the Rpn value will drop to 0 or even a negative number. It can be seen from Fig. 1c that there are typically positive and negative characteristic absorption peaks at $220 \mathrm{~nm}$ and $197 \mathrm{~nm}$ before and after the CF modification. According to calculations, the Rpn values of CF and CFOB-10 are 0.183 and 0.161 respectively, indicating that they retain a complete triple helix structure. It is worth noting that the intensity of the positive absorption peak after modification is slightly reduced, the intensity of the negative absorption peak is slightly increased, and the Rpn value is reduced, but the change is not large compared with the overall peak intensity. This may be due to the Schiff base reaction between the active groups on the side chain of collagen and the aldehyde groups on OBSP.

\subsection{Differential scanning calorimetry (DSC)}

The thermal denaturation temperature of collagen can reflect the stability of its triple helix structure to a certain extent and has an important influence on the subsequent processing and molding of collagen and the storage and transportation of products. This temperature is usually measured by differential scanning calorimetry (DSC). Generally, there are two characteristic peaks in the DSC detection graph of collagen, as shown in Fig. 1d. As the temperature gradually increased, the microstructure and macroscopic properties of collagen gradually changed. When the thermal denaturation temperature of collagen was reached, the three-strand helix structure of collagen was completely untwisted and dissociated into three randomly coiled $\alpha$ polypeptide chains, and the many excellent biological properties of collagen would also be weaken or even disappear completely [17]. It can also be seen from Fig. 1d that with the increasing amount of OBSP, the thermal denaturation temperature of $\mathrm{CF}$ increases from $71.6{ }^{\circ} \mathrm{C}$ to $82.7{ }^{\circ} \mathrm{C}$, which indicates that the Schiff base reaction between CF and OBSP can significantly improve the stability of CF. In addition, due to the formation of hydrogen bonds between a large number of carboxyl groups on the molecular chain of OBSP and amino groups and carboxyl groups on the collagen side chain, this is also conducive to the improvement of the thermal stability of CF. Although the original intention of the synthesis of OBSP is to improve the hemostatic performance, the increase in the thermal denaturation temperature shown by DSC proves that CFOB has better thermal stability than CF. Therefore, CFOB has a wider application potential and can be applied to more complex and extreme hemostasis scenarios.

\subsection{AFM and SEM}

An anatomic force microscope was used to observe the morphological structure of CFOB, as shown in Fig. 2. The fiber of pure collagen is uniform in thickness and has a neatly arranged structure, which is arranged in a network as a whole, but the woven structure between the CF is relatively loose. The cross-linked collagen fiber structure is relatively rough, and the order of the fiber is significantly reduced, and with the increase of the amount of OBSP, it becomes rougher and more chaotic. OBSP introduced a dialdehyde structure, but still retained most of the structure of the BSP main chain, with a relatively large molecular weight. The aldehyde group of OBSP reacts with the side chain amino group of collagens to form a Schiff base covalent bond, thereby inserting the main chain structure of BSP into the collagen fiber. The introduction of OBSP enables the secondary bonds that maintain the regular arrangement of collagen molecules, such as hydrogen bonds, to be replaced by Schiff base covalent bonds with stronger reactivity. The more OBSP dosage, the more significant this effect will be. Due to the longer molecular chains of CF and OBSP, they form an intertwined structure, and its complexity is proportional to the amount of OBSP, which ultimately turns the fiber network structure of pure collagen into an irregular flocculent structure. The loose network structure formed by this two-component entanglement is conducive to promoting cell adhesion and material transmission.

It can be seen from Fig. 3 that as the amount of OBSP increases, the pore size of CFOB becomes smaller and the pores become more uniform. A large number of sheet-like and filamentous structures in the pure collagen sponge are changed to a more uniform pore-like structure, which enhances the absorption capacity of the composite sponge to blood. While better adhering to the wound surface, the sponge can absorb and aggregate platelets, provide an aggregation platform for platelets, and activate platelets to release coagulation factors and thrombin. The release of the two will induce the conversion of fibrillin to form fibrin, and further promote the aggregation of fibrin monomers to form stable fibrin polymers, and finally produce clots, achieving the purpose of rapid and effective hemostasis.

\subsection{Determination of water absorption and porosity ratio}

The larger porosity can provide more space for the hemostatic material to absorb the blood on the wound surface so that CFOB can better play a hemostatic effect. At the same time, the larger porosity makes the sponge have stronger physical adsorption and can better adhere to the wound surface. Therefore, as an ideal hemostatic sponge, the porosity of CF is usually required to reach more than 
(a)

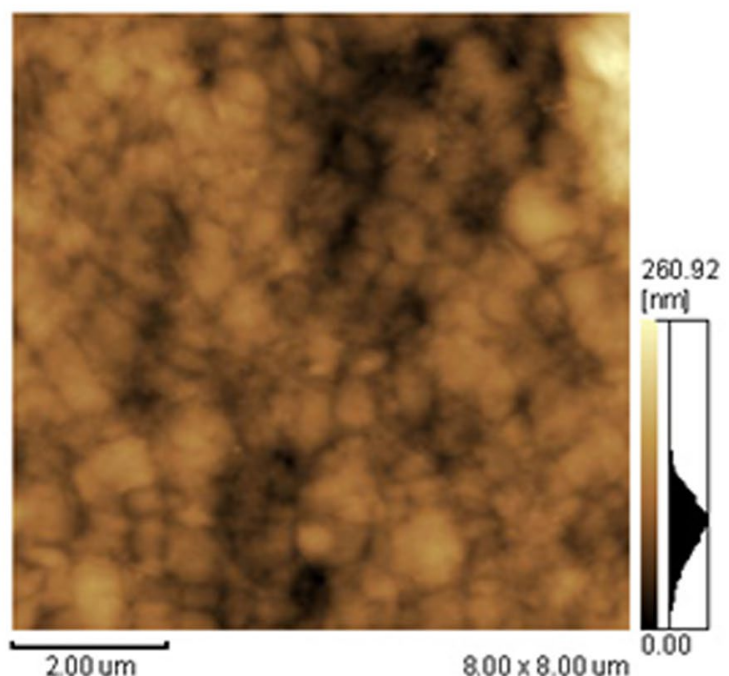

(c)

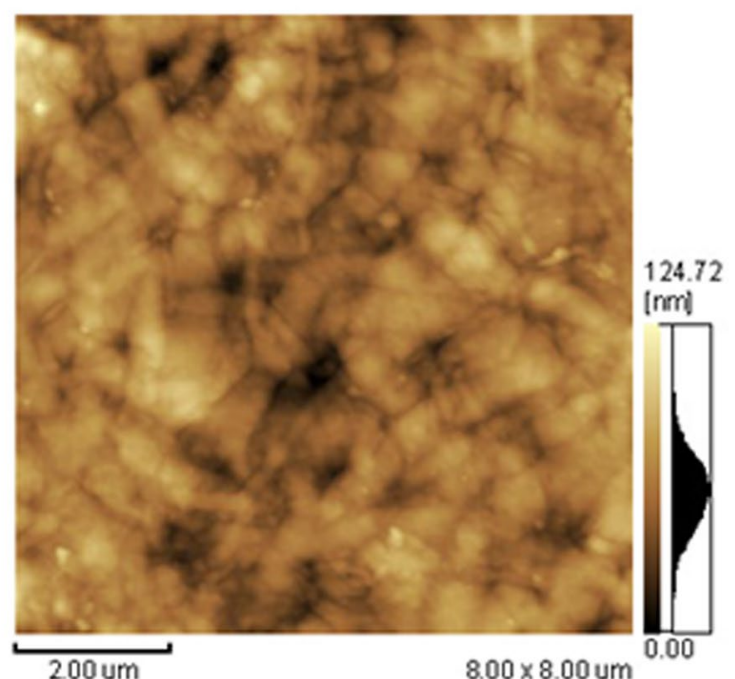

(b)

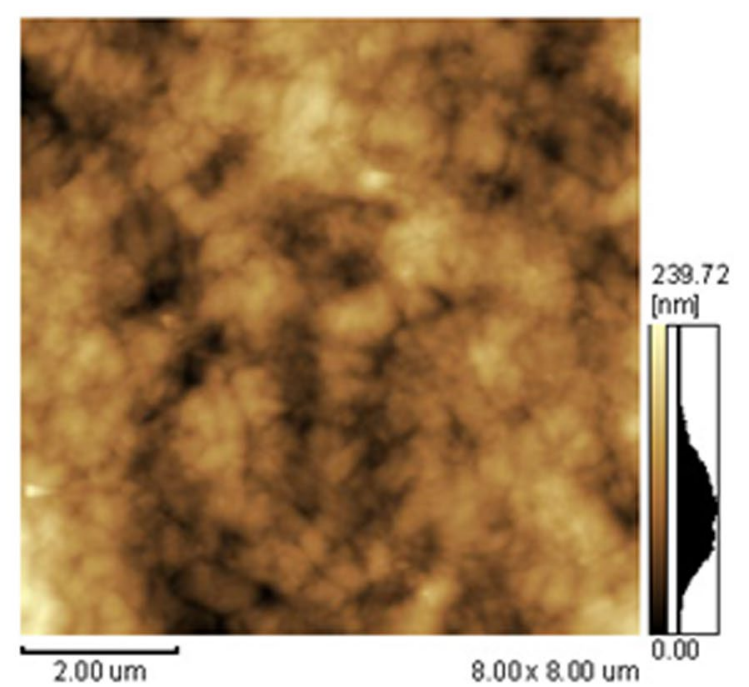

(d)

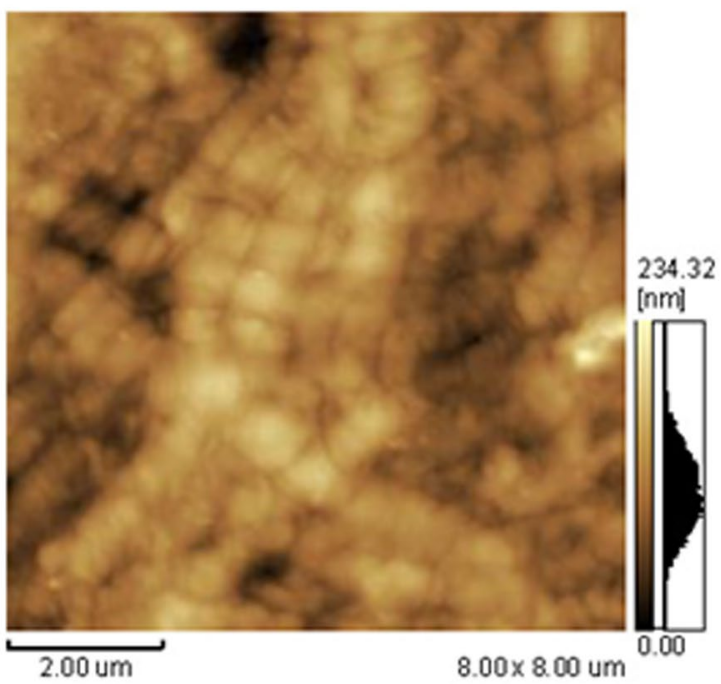

Fig. 2 AFM image of CFOB with different OBSP dosages: a CFOB-0, b CFOB-2, c CFOB-15, d CFOB-20

90\%. It can be seen from Fig. 4a that no matter how the content of OBSP changes, the porosity of CFOB is always maintained above $97 \%$, and the extremely high bulkiness meets the requirements of ideal hemostatic materials.

It can be seen from Fig. 4b that as the content of OBSP increases, the water absorption of CFOB first increases and then decreases. This may be because a small amount of OBSP consumes fewer hydrophilic groups of CF, while OBSP provides a large number of hydrophilic groups, which increases the water absorption of the sponge. However, when too much OBSP is introduced, a large amount of the hydrophilic groups of the CF itself is consumed, and the porosity of the sponge decreases, resulting in a decrease in water absorption. Comprehensive analysis shows that when the content of OBSP is $10 \%$ of $\mathrm{CF}$, the sponge has the best water absorption. On the whole, no matter how the amount of OBSP is changed, the CF sponge has a superior water absorption rate and can show a satisfactory liquid absorption effect.

\subsection{Hemolysis assay}

The hemolysis rate is related to the safety of the material. Hemolysis is a phenomenon in which red blood cells are destroyed and hemoglobin is released. When 

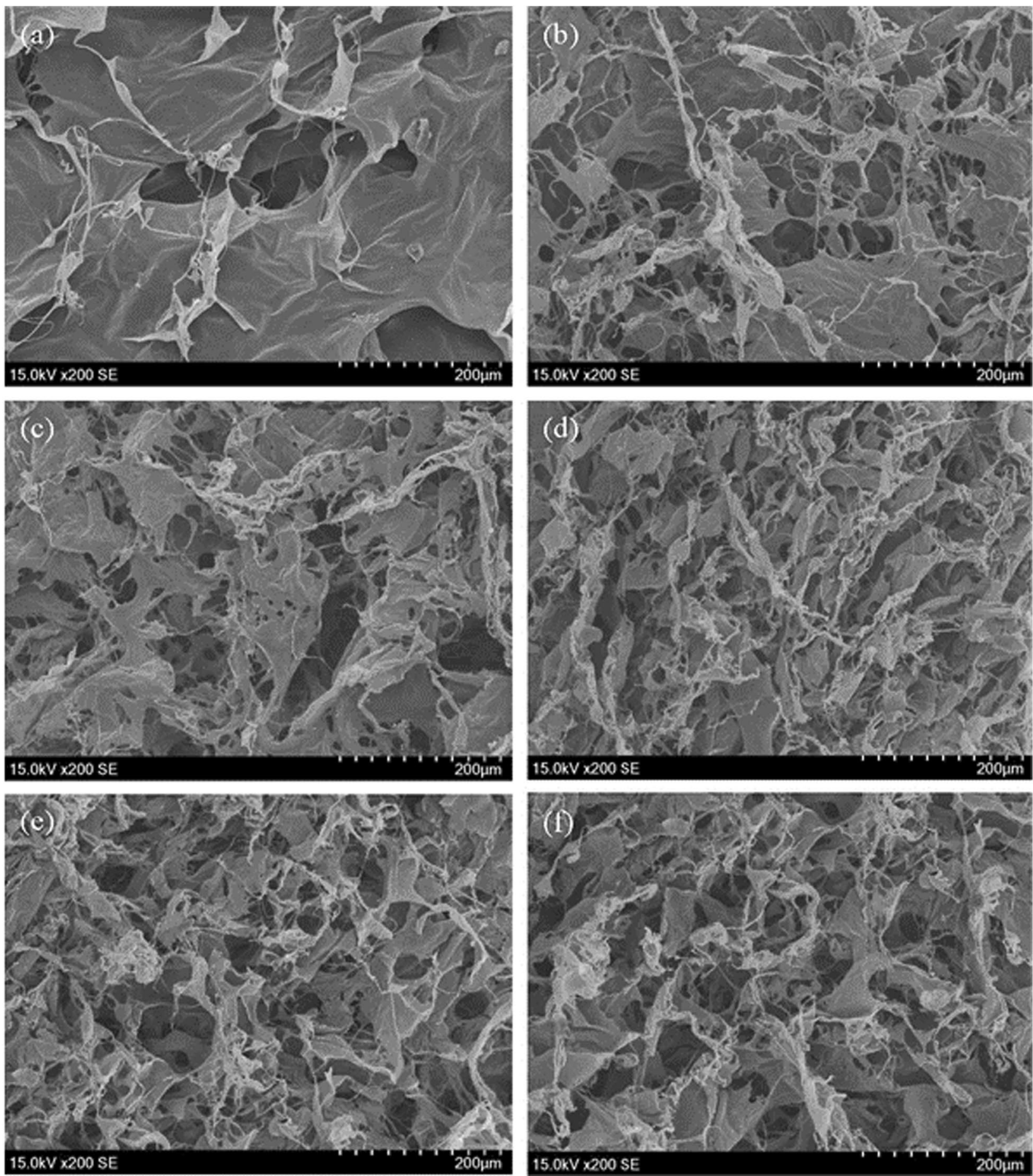

Fig. 3 SEM image of CFOB with different OBSP dosages: a CFOB-0, b CFOB-2, c CFOB-6, d CFOB-10, e CFOB-15, f CFOB-20

intense hemolysis occur, it will certainly harm the organism. Generally, the hemolysis rate of hemostatic materials below $5 \%$ stay in the safety. It can be seen from Fig. $4 \mathrm{c}$ that as the amount of OBSP increases, the hemolysis rate of
CFOB grows to a certain extent, but even the sample with the highest hemolysis rate does not exceed $1.4 \%$. Generally speaking, $\mathrm{CFOB}$ all possesses extremely low damage to red blood cells, meeting the requirements of medical materials. 


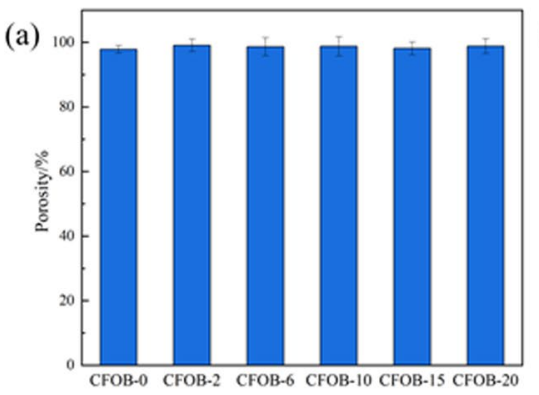

(b)

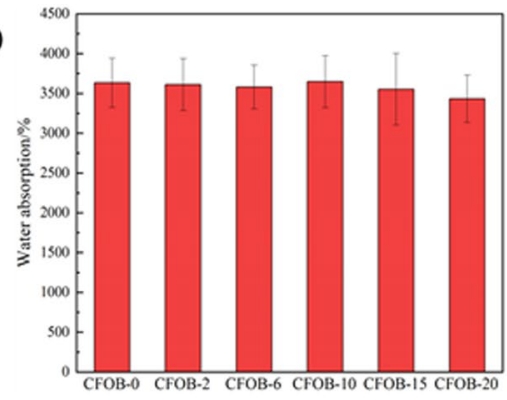

(c)

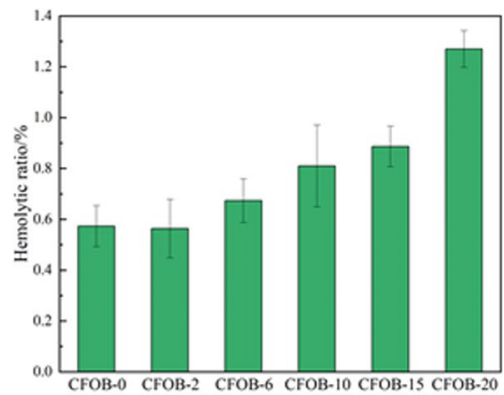

(d)

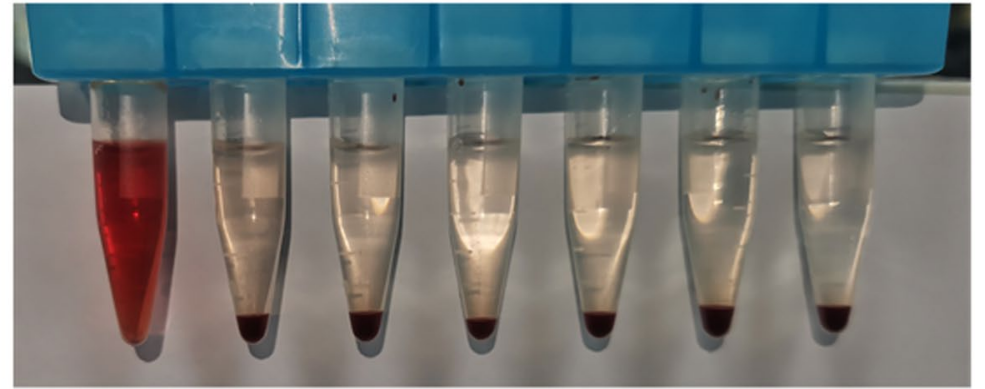

Fig. 4 a Porosity determination chart of CFOB with different OBSP dosages, $\mathbf{b}$ water absorption determination chart of CFOB with different OBSP dosages, $\mathbf{c}$, $\mathbf{d}$ hemolysis rate of CFOB with different OBSP dosages

\subsection{Activated partially thromboplastin time (APTT), prothrombin time (PT) and thrombin time (TT)}

APTT, PT, and TT are indicators of in vitro clotting. Among them, TT is mainly related to comprehensive factors such as plasma fibrinogen level, coagulation activity, and fibrin content, and its length can reflect the level of the common pathway of material-inducing coagulation factors. PT is an important indicator of the exogenous coagulation system. The length of PT is closely related to the levels of fibrinogen, prothrombin, and coagulation factor V, factor VII, and factor X in plasma. APTT is the phospholipid plasma recalcification time, which can describe the levels of fibrinogen, prothrombin, and coagulation factor $\mathrm{V}$ and factor $\mathrm{X}$ in the common pathway of coagulation factors of the endogenous coagulation system in plasma.

According to the APTT, PT, and TT detection diagrams shown in Fig. 5, it is not difficult to find that with the addition of OBSP, the APTT and TT values gradually decrease, which indicates that CFOB play an significant role in the physiological coagulation system: OBSP not only activates the expression of coagulation factor VI, factor IX, factor XI, and factor XII, accelerating the initiation of the endogenous coagulation system, but also promotes the activation of coagulation factor III and factor VII in the exogenous coagulation system. On the contrary, there is no obvious rule and no significant difference in the reaction time of $\mathrm{PT}$. That is, during the whole coagulation process, it is through endogenous coagulation that hemostasis of CFOB is to shorten the blood coagulation time, not the exogenous coagulation system. In this process, CF and BSP can promote the release of coagulation factor XII from platelets, and activate factor XII to factor XII-a, thereby starting the entire endogenous coagulation and shortening the clotting time.

\subsection{Evaluation of cell compatibility}

Good biocompatibility is one of the necessary conditions for medical materials. It can be seen from Fig. 6 that CFOB has almost no effect on the proliferation of fibroblasts. When the dosage of OBSP was increased from 0 to $15 \%$ of $\mathrm{CF}$, the cells relative proliferation rate of the samples was all above $80 \%$, and they met the requirements of level 0 in the cytotoxicity classification standard. Due to the limited number of free amino groups on CF, when the amount of OBSP is less than $10 \%$ of CF, a large number of amino groups in CF can react better with aldehyde groups. The greater the consumption of aldehyde groups are, the lower the residual amount is. When the dosage of OBSP is increased continuously, the amino group of CF gradually reacts completely. Excessive aldehyde groups cannot react with amino groups, resulting in an increase in the number of free aldehyde groups and unfavorable for cell growth. Especially when the amount 
(a)

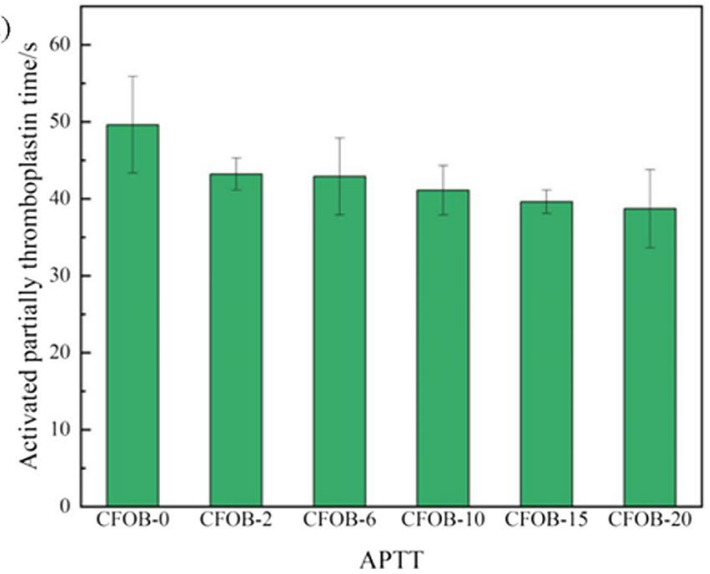

(b)

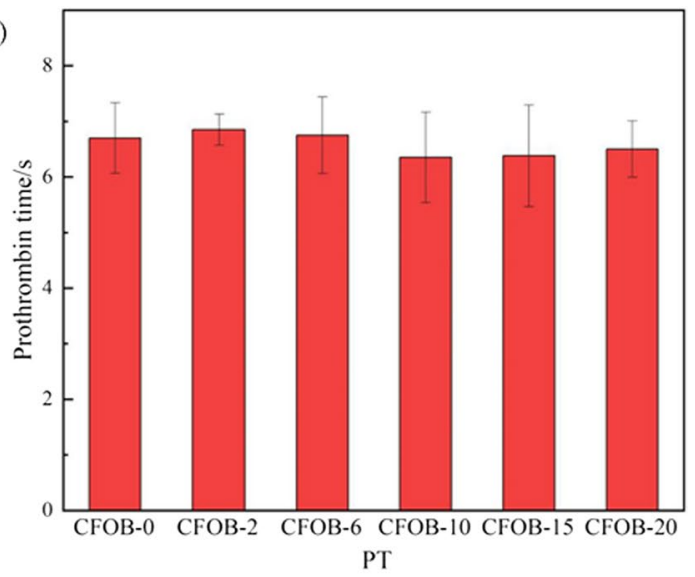

(c)

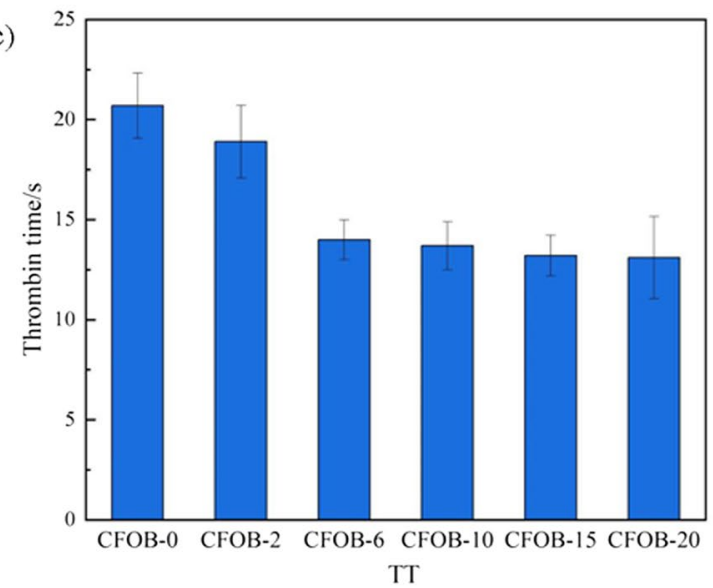

Fig. 5 a APTT, b PT, and $\mathbf{c}$ TT of CFOB with different OBSP dosages

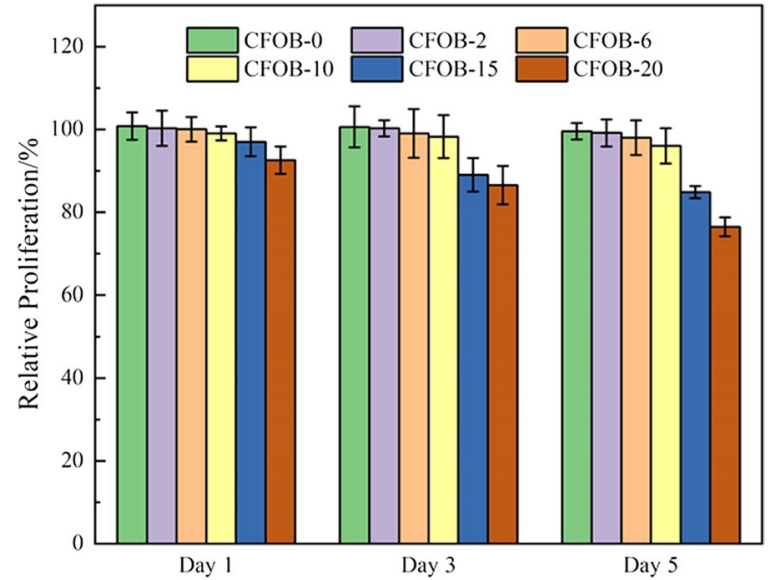

Fig. 6 CCK-8 experiment results of CFOB with different OBSP dosages

of OBSP is more than $15 \%$ of CF, the free amino groups on the CF have reacted completely. Excessive OBSP leads to a relative growth in the dosage of residual aldehyde groups, and they greatly reduce the cell proliferation rate. Therefore, when the cell activity of OBSP-10 is the best.

\subsection{Whole-blood clotting assay (CT)}

Whole-blood clotting time $(\mathrm{CT})$ is mainly related to the content and function of various types of coagulation factors in the blood, coagulation factors, and the activity of anticoagulation factors, and can directly reflect the hemostatic properties of materials in vitro [18].

It can be seen from Fig. 7a that when $0.2 \mathrm{ml}$ of fresh anticoagulated rat blood was added, all-composite hemostatic materials with different contents achieved hemostasis within $1 \mathrm{~s}$. When $0.4 \mathrm{ml}$ of fresh anticoagulated rat blood was added, there were some differences, but sponges with OBSP content of $10 \%$ of CF or above still stopped bleeding in $1 \mathrm{~s}$. Yet, $1 \mathrm{~s}$ bleeding time was helpless in reflecting the hemostatic effect of the contents of $\mathrm{CFOB}$, so we involved an increased experimental blood volume to $0.6 \mathrm{mg}$. When $0.6 \mathrm{ml}$ of fresh anticoagulant rat blood was added, the hemostatic performance of CFOB was significantly better than that of 
(a)

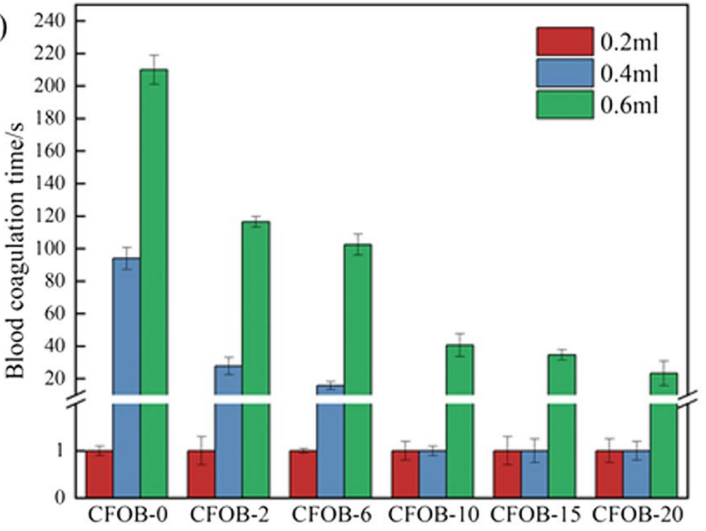

(b)

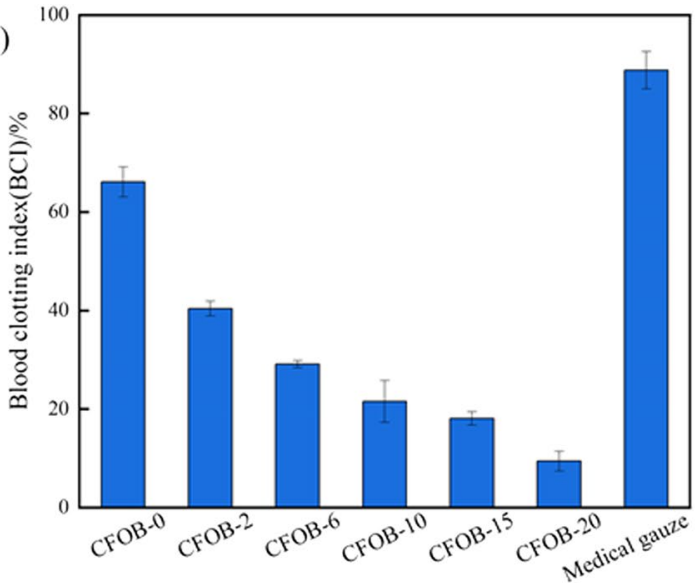

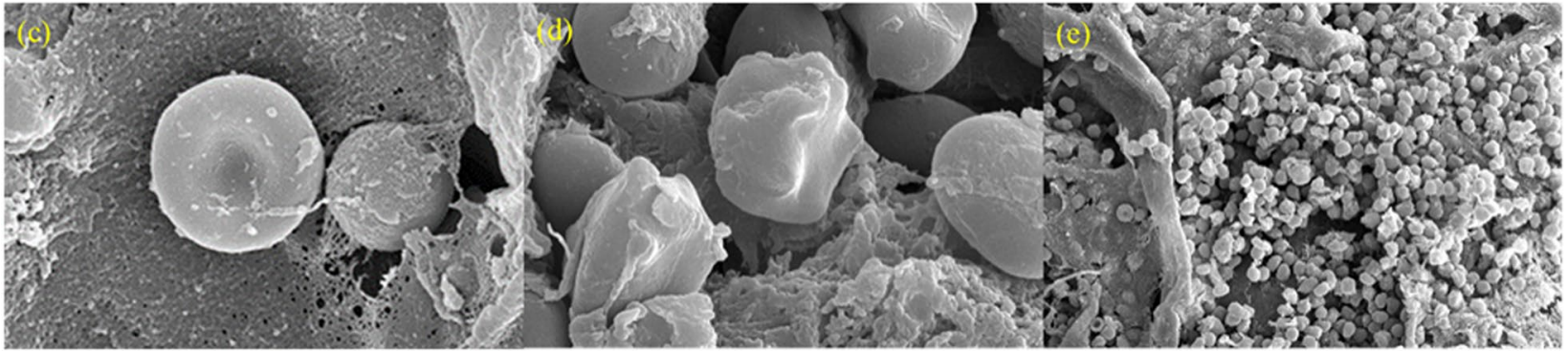

Fig. 7 a Blood coagulation time of CFOB with different OBSP dosages, $\mathbf{b}$ BCl of CFOB with different OBSP dosages, the observation of blood cells andmaterial interaction via electron microscope: $\mathbf{c}$ at $1 \mathrm{~min}$, red blood cells adhere to the surface of CFOB, 10,000 $\times$, d At 3 min, red blood cells expand and expand pseudopodia accompanied by aggregation and adhesion to CFOB sponge, 10,000 x, e at 5 min, blood cells begin to aggregate in large numbers, $1000 \times$

pure $\mathrm{CF}$, which indicated that the endogenous coagulation factors of CF modified by OBSP were activated to a higher degree. And as the content of OBSP increased, the hemostatic performance also became superior, and CFOB-20 showed the shortest hemostatic time at about 20 s. Compared with pure CF, there are more hydrophilic groups on OBSP, so fresh anticoagulated rat blood is easier to penetrate the material faster. In addition, materials with smaller pore diameters are also easier to adsorb platelets, thereby forming thrombus and promoting the release of clotting factors. In the traditional method, the physical and chemical properties of collagen crosslinked with glutaraldehyde have been greatly improved, but the introduction of cross-linking agents reduces the hydrophilic groups of the collagen molecular chain, and an ultimately decrease in the hydrophilicity and significant elongation of the hemostatic time confirm this point. The biocompatibility modified by glutaraldehyde is difficult to meet clinical requirements. Therefore, in terms of hemostasis and biocompatibility, the new OBSP crosslinker is superior to the traditional glutaraldehyde crosslinker.

\subsection{Blood clotting assay in vitro}

The blood-clotting index $(\mathrm{BCI})$ can reflect the in vitro coagulation performance of the material. Different from the total clotting time, BCI can reflect the ability of the hemostatic material to form a thrombus after contact with blood. The lower BCI stands, the better hemostatic performance shows. Figure $7 \mathrm{~b}$ demonstrates that the effect of different OBSP dosages brings huge influence to the $\mathrm{BCI}$ of the composite hemostatic material. It can be found that the hemostatic effect of CFOB is beyond medical gauze. The modified CF has a stronger ability to form thrombus after contact with blood, and the main component of thrombus is platelets, which can predict that its ability to promote platelet adhesion and aggregation may be stronger. As the dosage of OBSP continues to increase, the number of $\mathrm{BCI}$ continues to decrease, that is, CFOB20 expresses the best hemostasis ability among them, and the average coagulation index remains $9.41 \pm 2.02 \%$. It is worth mentioning that the $\mathrm{BCI}$ of $\mathrm{CFOB}$ with an OBSP content of more than $10 \%$ of CF is lower than $20 \%$, which is better than some hemostatic materials reported in the literature [19]. 
Scanning Electron Microscopy was used to observe the adhesion and morphological changes of blood cells as well as platelets on the surface of the material. At $1 \mathrm{~min}$ after adding blood into CFOB like Fig. 7c shows, it was observed that red blood cells and platelets were distributed on the material surface with good morphology, and adsorbed by material, which showed the beneficial internal blood compatibility and spatial structure of the material. The morphology change of red blood cells was occurred with the appearance of stretched pseudopodia among cells at $3 \mathrm{~min}$, implying the physiological changes of cells including aggregation and deformation like Fig. $7 \mathrm{~d}$ shows. At $5 \mathrm{~min}$, Blood cells continue to accumulate in CFOB in large numbers like Fig. 7e shows.

\subsection{Evaluation of in vivo hemostatic performance}

Therefore, to visually compare the hemostatic effect, the bleeding time and blood loss of the wound were used as indicators to analyze and compare the hemostatic performance of the following three materials in animals: medical gauze, unmodified collagen fiber sponge (CF), and CFOB. After considering the hemostatic performance and cytotoxicity, CFOB-10 was selected for animal experiments. Figure $8 \mathrm{a}, \mathrm{b}$ show the hemostatic time, hemorrhage volume, and hemostatic effect of these materials in the rat liver trauma hemorrhage model. It can be seen that the amount of bleeding decreased from $126.67 \pm 19.11 \mathrm{mg}$ of the blank control group to $23.5 \pm 4.95 \mathrm{mg}$ of self-made CFOB-10, which is also less than the hemostatic volume of medical gauze $70.33 \pm 15.51 \mathrm{mg}$ and the hemostatic volume of CF $35.67 \pm 10.505 \mathrm{mg}$. The clotting time of each material was $150.4 \pm 29.555 \mathrm{~s}$ for blank control, $73 \pm 19 \mathrm{~s}$ for medical gauze, $50.33 \pm 4.51 \mathrm{~s}$ for $\mathrm{CF}$, and $25 \pm 4.06 \mathrm{~s}$ for CFOB-10. The hemostatic time of CFOB was also greatly reduced. Figure $8 \mathrm{c}, \mathrm{d}$, e also shows the hemostatic effect and time comparison of the blank group and CFOB. When the wound with CFOB was used for hemostasis, there was no blood to ooze out, the blank group continued to ooze blood. According to Liu [20], CF is woven into $\mathrm{CF}$ through the accumulation, aggregation, arrangement, and entanglement of collagen molecules. The hemostatic properties of CF are enhanced with the increase in the number of layers. Therefore, CFOB is

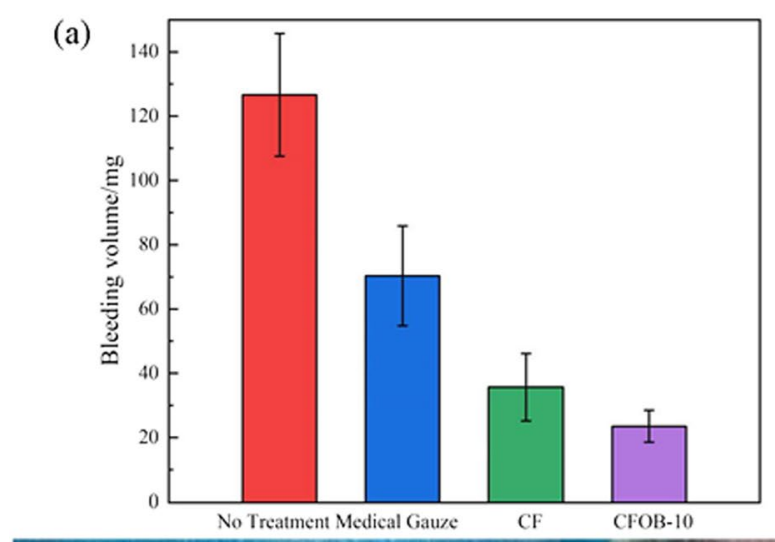

(b)
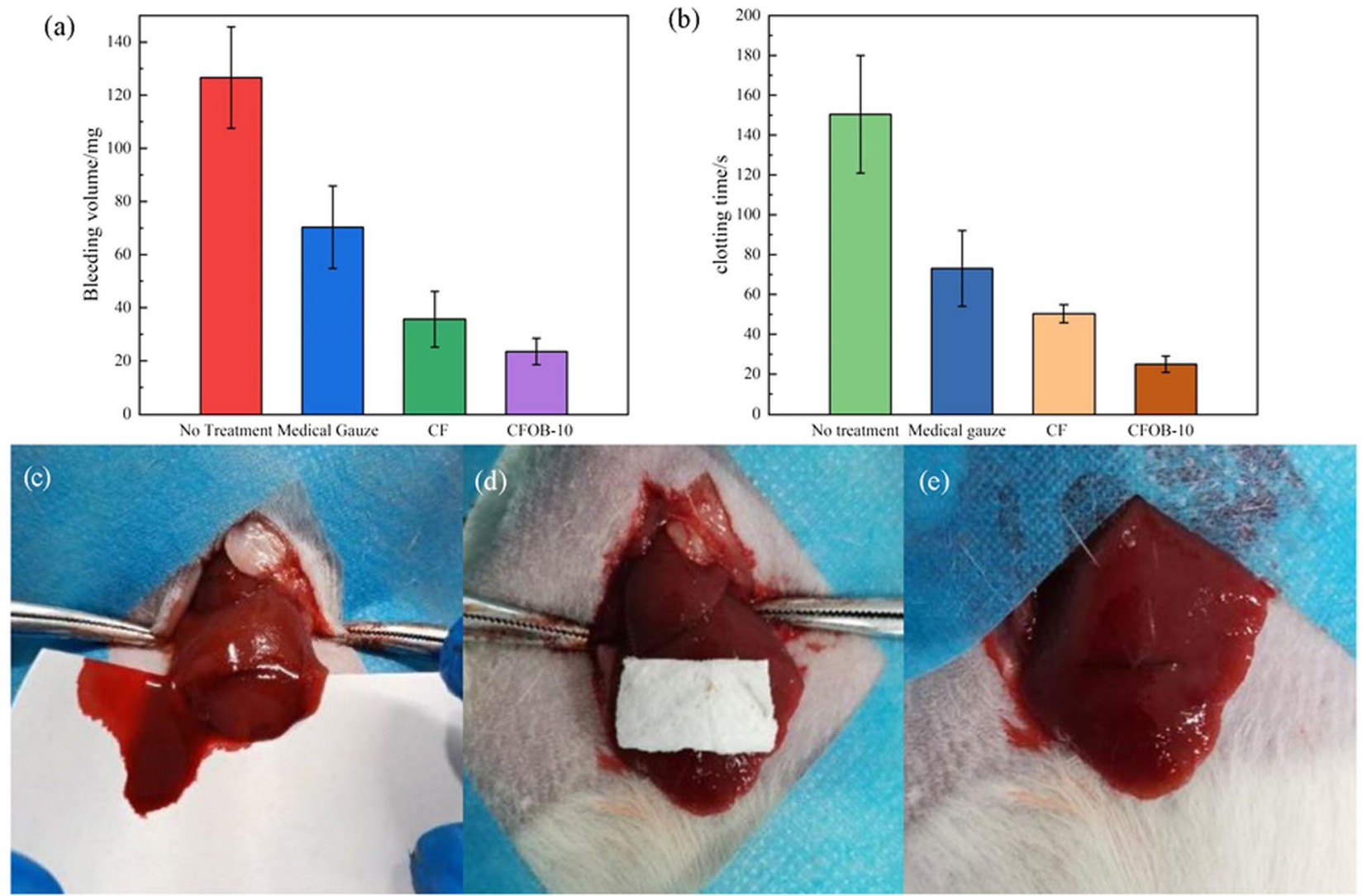

Fig. 8 Comparison of $\mathbf{a}$ blood loss and $\mathbf{b}$ clotting time in animal experiments with different materials, The comparison of the hemostatic effect of c the blank control group and $\mathbf{d}$ and $\mathbf{e}$ CFOB at the same time is shown. There was no oozing in the CFOB wound when hemostasis, and the blank group continued to ooze 
significantly better than collagen fiber sponge, collagen sponge, and medical absorbable gauze in terms of bleeding volume and hemostasis time. At the same time, CFOB can specifically recognize and adhere to platelets, and it can promote the activation of related coagulation factors and have better hemostatic performance.

\subsection{Analysis of coagulation mechanism and synergy}

The hemostasis process of the body is achieved through the triple physiological reactions of vasoconstriction, hemostasis thrombosis, and blood coagulation system, all of which impact and control each other. Blood vessels are prompted to release angiotensin and other chemicals to speed up vasoconstriction when the body is injured. After that, the vascular wall tissue factor specifically recognizes platelet membrane integrin GPVI, activates platelet adhesion, aggregation, and release of procoagulant factors, forming preliminary hemostasis of thrombosis. At the same time, the blood coagulation system is activated, various types of coagulation factors are activated hierarchically, and the coagulation factors activate prothrombin. The activated prothrombin can catalyze the conversion of fibrinogen into aggregated fibrin to strengthen the thrombus to achieve secondary hemostasis. According to the electron microscope observation picture in Fig. 7c, d, e, the aggregation and adhesion process of blood cells can be seen.

However, severe bleeding often occurs in emergencies, the time for hemostasis is tight and the conditions are limited, resulting in the body's inability to complete the hemostasis process, which often affects life safety. CF has good biocompatibility and hemostatic properties and is often made into a sponge hemostatic agent with easy operation and good water absorption to respond to emergencies. CF locally stimulates the adhesion and aggregation of platelets and activates the coagulation system to produce coagulation factors and thrombin, thereby inducing the conversion of fibrillin into fibrin and further aggregation to form fibrin polymer, and finally produce a clot to achieve a rapid and effective hemostatic effect [2]. The complexity of the coagulation mechanism determines that the hemostatic performance of CF can only meet the basic requirements, but still cannot cope with more changes. BSP, extracted from a natural herb Bletilla striata, has a blood clotting mechanism similar to CF. According to the report of Kaplani and colleagues [21], BSP was able to promote platelet aggregation and activate the coagulation factors of the endogenous and exogenous coagulation pathways to achieve hemostasis. Therefore, the two should have synergy on wound hemostasis. However, only the mechanically mixing of CF and BSP has shortcomings such as easy delamination, which cannot fundamentally improve the material properties.
Therefore, we used the natural herbaceous plant Bletilla striata as the raw material to extract the effective ingredient BSP and oxidize it. FT-IR detection proved that BSP was successfully oxidized to OBSP by sodium periodate under dark conditions. The extracted beef tendon source CF and OBSP underwent Schiff base reaction to obtain CFOB. CD detection and FT-IR detection together proved that the modification process did not destroy the triple helix structure of collagen. In the characterization experiment, AFM, SEM, and DSC tests jointly proved that the adsorption, material transport capacity, and thermal stability of CFOB increase with the increase of OBSP dosage. The porosity test and hemolysis rate test proved that all amounts of CFOB had good porosity and blood compatibility. Through water absorption test, cytotoxicity test, and in vitro coagulation index APTT, PT, TT, CT, BCI, etc., CFOB10 with the best hemostasis performance was screened, that is, the optimal dosage was OBSP $0.5 \mathrm{mg} / \mathrm{mL}, \mathrm{CF}$ $5 \mathrm{mg} / \mathrm{mL}$. Applying the best-performing CFOB-10 to the rat liver hemorrhage model, it could be seen that CFOB-10 had a better hemostatic performance than medical gauze and CF. The bleeding volume and hemostasis time were reduced to $23.5 \pm 4.95 \mathrm{mg}$ and $25 \pm 4.06 \mathrm{~s}$, respectively. It also indirectly proved the hemostatic synergistic effect of OBSP and CF.

\section{Conclusions}

The freeze-dried sponge CFOB was created by crosslinking CF and OBSP, and it preserved the outstanding structure and biological properties of CF. The various features of $C F O B$ were superior to $C F$, especially DSC findings and in vitro coagulation experiments, which demonstrated that CFOB's thermal stability and coagulation ability had been improved. In the cytotoxicity test, the biocompatibility of CFOB below the specific OBSP dosage met the 0 level requirements of the cytotoxicity classification standard. Finally, in vivo animal experiments proved that CFOB had good hemostatic characteristics. Based on the above experimental results, the best ratio of CFOB with good thermal stability, biocompatibility, and excellent hemostatic properties, shows extremely high clinical application potential.

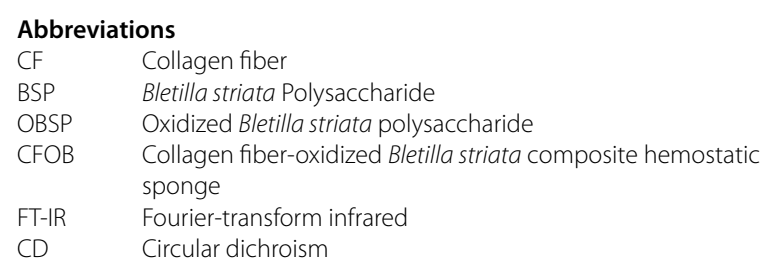




$\begin{array}{ll}\text { DSC } & \text { Differential scanning calorimetry } \\ \text { AFM } & \text { Atomic force microscopy } \\ \text { SEM } & \text { Scanning electron microscopy } \\ \text { APTT } & \text { Activated partially thromboplastin time } \\ \text { PT } & \text { Prothrombin time } \\ \text { TT } & \text { Thrombin time } \\ \text { BCI } & \text { Blood clotting index } \\ \text { CCK-8 } & \text { Cell Counting Kit-8 }\end{array}$

\section{Supplementary Information}

The online version contains supplementary material available at https://doi. org/10.1186/s42825-022-00079-2.

Additional file 1: Supplementary experiments.

Additional file 2: Video material of the rat liver hemorrhage model experiment.

\section{Acknowledgements}

We thank the Opening Project of Key Laboratory of Leather Chemistry and Engineering, (Sichuan University), Ministry of Education, (SCU2021D005) and the Fundamental Research Funds for the Central Universities (20826041E4156 and 20826041(4159).

\section{Authors' contributions}

XY: Conceptualization, Methodology, Investigation, experiments, Writingoriginal draft \& editing. YC: Conceptualization, Methodology, Writing-review. WD: Methodology. ND: Conceptualization, Methodology, Supervision. ZL: Methodology. All authors read and approved the final manuscript.

\section{Funding}

We acknowledge the financial support provided by Opening Project of Key Laboratory of Leather Chemistry and Engineering, (Sichuan University), Ministry of Education, (SCU2021D005) and the Fundamental Research Funds for the Central Universities (20826041E4156 and 20826041C4159).

\section{Availability of data and materials}

All data generated or analyzed during this study are included in this manuscript and Additional files 1 and 2.

\section{Declarations}

\section{Ethics approval and consent to participate}

The animal study was approved by the Ethical Committee of Sichuan University (20221116004). All the animals were purchased from Laboratory Animal Center of Sichuan University and the animal certification is No. SCXK (Chuan 2018-26). The animal experiment guidance from the ethical committee and the guide for care and use of laboratory animals from $\mathrm{NIH}$ were followed during the whole experiment course.

\section{Competing interests}

The authors declare that they have no competing interests.

\section{Author details}

${ }^{1}$ Department of Biomass Science and Engineering, Sichuan University, Chengdu 610065, People's Republic of China. ${ }^{2}$ National Engineering Research Center of Clean Technology in Leather Industry, Sichuan University, Chengdu 610065, People's Republic of China. ${ }^{3}$ The Key Laboratory of Leather Chemistry and Engineering of Ministry of Education, Sichuan University, Chengdu 610065, People's Republic of China.

Received: 8 August 2021 Accepted: 21 January 2022

Published: 15 February 2022

\section{References}

1. Hamada SR, Rosa A, Gauss T, Desclefs JP, Raux M, Harrois A, Follin A, Cook F, Boutonnet M, Attias A. Development and validation of a pre-hospital
"Red Flag" alert for activation of intra-hospital haemorrhage control response in blunt trauma. Crit Care. 2018;22(1):113.

2. Manon-Jensen T, Kjeld NG, Karsdal MA. Collagen-mediated hemostasis. J Thromb Haemost. 2016;14(3):438-48.

3. Zhang Z, Ma Z, Zhang Y, Chen F, Zhou Y, An Q. Dehydrothermally crosslinked collagen/hydroxyapatite composite for enhanced in vivo bone repair. Colloids Surf B Biointerfaces. 2018;163:394-401.

4. Jiang X, Wang Y, Fan D, Zhu C, Liu L, Duan Z. A novel human-like collagen hemostatic sponge with uniform morphology, good biodegradability and biocompatibility. J Biomater Appl. 2017;31(8):1099-107.

5. Zhang C, He Y, Chen Z, Shi J, Qu Y, Zhang J. Effect of polysaccharides from Bletilla striata on the healing of dermal wounds in mice. Evid Based Complement Alternat Med. 2019;2019:9212314.

6. Zhao L, Sun D, Lu H, Han B, Zhang G, Guan Q. In vitro characterization of pHsensitive Bletilla Striata polysaccharide copolymer micelles and enhanced tumour suppression in vivo. J Pharm Pharmacol. 2018;70:797-807.

7. Ju HY. Study on type I collagen fiber of bovine tendon source and its composite membrane material. Sichuan University. 2015.

8. Qu Y, Li C, Zhang C, Zeng R, Fu C. Optimization of infrared-assisted extraction of Bletilla striata polysaccharides based on response surface methodology and their antioxidant activities. Carbohyd Polym. 2016;148:345-53.

9. Kumar PS, Lakshmanan VK, Anilkumar TV, Ramya C, Reshmi P, Unnikrishnan AG, Nair SV, Jayakumar R. Flexible and microporous chitosan hydrogel/ nano $\mathrm{ZnO}$ composite bandages for wound dressing: in vitro and in vivo evaluation. Acs Appl Mater Interfaces. 2012;4(5):2618-29.

10. He Y, Wang J, Si Y, Wang $X$, Zhao J. A novel gene recombinant collagen hemostatic sponge with excellent biocompatibility and hemostatic effect. Int J Biol Macromol. 2021;178(2):296-305.

11. Eren SE, Karakukcu C, Ciraci MZ, Ustundag Y, Karakukcu M. Activated partial thromboplastin time derivative curves: helpful diagnostic tool in mixing test interpretation. Blood Coag Fibrinol. 2018;29(4):1.

12. Jin J, Ji Z, Xu M, Liu C, Ye X, Zhang W, Li S, Wang D, Zhang W, Chen J, Ye F, Lv Z. Microspheres of carboxymethyl chitosan sodium alginate, and collagen as a hemostatic agent in vivo. ACS Biomater Sci Eng. 2018;4(7):2541-51.

13. Mu C, Guo J, Li X, Wei L, Li D. Preparation and properties of dialdehyde carboxymethyl cellulose crosslinked gelatin edible films. Food Hydrocolloids. 2012;27(1):22-9.

14. Andrews ME, Murali J, Muralidharan C, Madhulata W, Jayakumar R. Interaction of collagen with corilagin. Colloid Polym Sci. 2003;281(8):766-70.

15. Dan W, Chen Y, Dan N, Zheng X, Wang L, Yang C, Huang Y, Liu X, Hu Y. Multi-level collagen aggregates and their applications in biomedical applications. Int J Polymer Anal Charact. 2019;24(8):667-83.

16. Liu XH, Dan NH, Dan WH, Gong JX. Feasibility study of the natural derived chitosan dialdehyde for chemical modification of collagen. Int J Biol Macromol. 2016;82:989-97.

17. Hu Y, Jiang YG, Dan WH, Liu L, Lin H, Dan NH, Mi ZJ. Pepsin extraction of pig skin collagen. China Leather. 2010;39(023):11-6.

18. Sabino RM, Popat KC. Evaluating whole blood clotting in vitro on biomaterial surfaces. Bio-Protoc. 2020;10(3): e3505.

19. Jin J, Ji Z, Xu M, Liu C, Ye X, Zhang W, Li S, Wang D, Zhang W, Chen J, Ye F, Lv Z. Microspheres of carboxymethyl chitosan, sodium alginate, and collagen as a hemostatic agent in vivo. ACS Biomater Sci Eng. 2018:4(7):2541-51.

20. Liu XH. Preparation and performance of functional hemostatic materials based on high-level collagen aggregates. Sichuan University. 2017.

21. Kaplani K, Koutsi S, Armenis V, Skondra FG, Karantzelis N, Tsaniras SC, Taraviras S. Wound healing related agents: ongoing research and perspectives. Adv Drug Deliv Rev. 2018;129:242-53.

\section{Publisher's Note}

Springer Nature remains neutral with regard to jurisdictional claims in published maps and institutional affiliations. 